Received: 2019.07 .22 Accepted: 2019.10.28 Published: 2020.01 .23

\title{
Chordoma: A Case Report and Review of Literature
}

Authors' Contribution:

Study Design A

Data Collection B

Statistical Analysis C

Data Interpretation D

Manuscript Preparation E

Literature Search $F$

Funds Collection G

\author{
EF 1 Arish Noor \\ EF 1 Poorva Bindal \\ E 2 Miguel Ramirez \\ EF 3 James Vredenburgh
}

1 Internal Medicine, University of Connecticut, Farmington, CT, U.S.A.

2 Department of Radiology, St. Francis Hospital, Hartford, CT, U.S.A.

3 Department of Hematology Oncology, St. Francis Hospital, Hartford, CT, U.S.A.
Corresponding Author: Conflict of interest:
Arish Noor, e-mail: noor@uchc.edu

None declared

\begin{abstract}
Patient: Female, 68-year-old
Final Diagnosis: Chordoma

Symptoms: Hoarseness $\bullet$ neck pain $\bullet$ weakness

Medication: -

Clinical Procedure: $\quad-$

Specialty: Oncology

Objective: Unusual or unexpected effect of treatment

Background: Chordoma is rare, but aggressive bone tumor, primarily affecting the axial skeleton. Systemic chemotherapies are not effective against the tumor, and treatment primarily consists of surgical resection and radiation. Despite these treatment modalities, recurrence is common. Our case highlights the role of afatinib as an effective treatment option in such cases.

Case Report: We present case of 68-year-old female with chordoma, who underwent multiple surgical resections, radiotherapy session, and had course complication by disease progression on imatinib and local recurrence. She was eventually placed on afatinib with good effect.

Conclusions: This article discusses the effectiveness of afatanib as a treatment modality, along with diagnosis, histopathological features, associated genetic aberrations, currently available and upcoming treatment options. Special emphasis is placed on molecular targeted therapy, emerging immunotherapies and use of vaccination in this field.
\end{abstract}

\section{MeSH Keywords: $\quad$ Chordoma $\bullet$ Molecular Targeted Therapy $\bullet$ Proton Therapy}

Abbreviations:

MRI - magnetic resonance imaging; GERD - gastroesophageal reflux disease; CSF - cerebrospinal fluid; TKR - tyrosine kinase receptor; EGFR - epidermal growth factor; PI3K - Akt/phosphoinositide-3-kinase; mTOR - mammalian target of rapamycin; MAPK - mitogen-activated protein kinase; ERK - extracellular signal-regulated kinase pathway; JAK - Janus kinase; STAT - signal transducer activation of transcription; RECIST criteria - response evaluation criteria in solid tumors; CAR - chimeric antibody receptor; CSPG-4 - chondroitin sulfate proteoglycan-4

Full-text PDF: https://www.amjcaserep.com/abstract/index/idArt/918927 


\section{Background}

Chordoma is rare, but aggressive bone tumor, primarily affecting the axial skeleton. It has incidence reported at 0.08/100 000; $0.1 / 100000$ in males and $0.06 / 100000$ in females [1,2]. Systemic chemotherapies are not effective against the tumor, and treatment primarily consists of surgical resection and radiation. We present case of 68-year-old female with chordoma, who underwent multiple surgical resections, radiotherapy session, and had course complication by disease progression on imatinib and local recurrence. She was eventually placed on afatinib with good effect. This article highlights the effectiveness of afatanib as a treatment modality, and discusses the diagnosis, histopathological features, associated genetic aberrations, currently available and upcoming treatment options.

\section{Case Report}

A 68-year-old female with past medical history of gastroesophageal reflux disease (GERD), gallstones, renal stones, hypertension, hyperlipidemia, obesity, osteoarthritis, and osteoporosis, presented with initial symptoms of hoarseness and neck pain. Magnetic resonance imaging (MRI) was performed that showed a $5.5 \mathrm{~cm}$ mass in the prevertebral area extending from C3 through C6. The biopsy was consistent with chordoma, (Figures 1, 2). Her past surgical history was significant for cholecystectomy, total abdominal hysterectomy and oophorectomy for possible history of remote malignancy. Family history was significant for diabetes mellitus, hyperlipidemia, colon cancer, heart disease, stroke; while social history was negative for smoking, alcohol or drug use.

She underwent surgical debulking followed by CyberKnife treatment with stereotactic radiation in 2010-2011. The treatment consisted of $35 \mathrm{~Gy}$ administered in 5 fractions to the residual tumor from C4 through C6.

In 2012, she had a recurrence and underwent a C4-C5 laminectomy with excision of the extradural tumor. The repeat pathology was again consistent with chordoma, and treatment with imatinib was initiated. She was on imatinib from January 2014 till January 2015. After being on imatinib for a year, she was noted to have disease progression requiring further surgical debulking. She underwent complete gross total resection and the pathology was again consistent with chordoma. She subsequently developed neck pain and motor weakness with imaging evidence of increased tumor in the epidural space at the C4-C5 and C5-C6 levels, causing severe canal stenosis and cord compression, with the tissue mass extending posteriorly from the vertebral body. She underwent urgent decompression laminectomy for spinal cord compression, following which her neurological symptoms improved (Figure 3). She was then

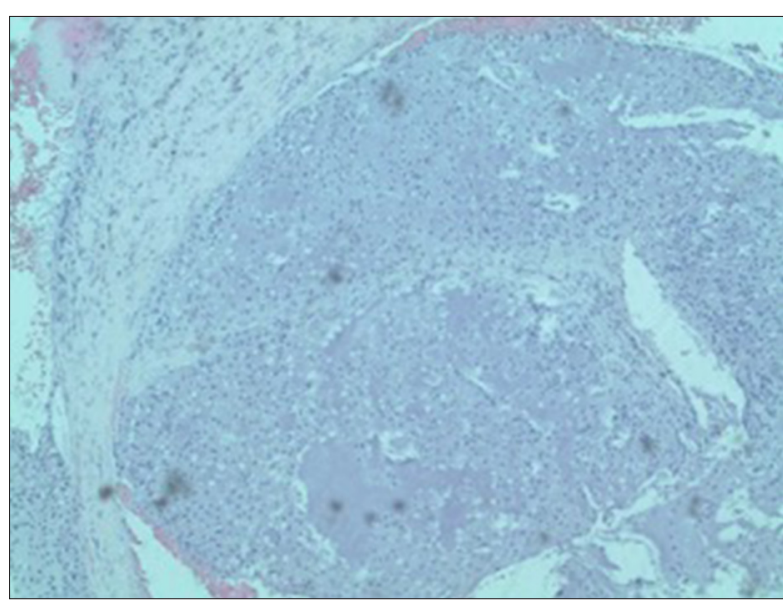

Figure 1. Low power magnification (50x) demonstrating lobular architecture and characteristic myxoid stroma.

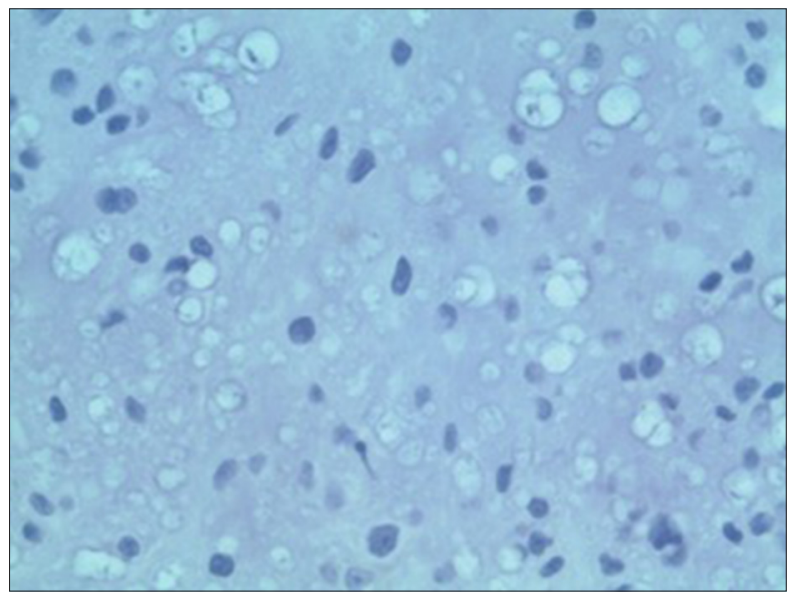

Figure 2. High power magnification (400x) showing cellular detail. Individual cells demonstrate a "bubby" cytoplasm (physaliphorous cells).

started on oral dexamethasone and afatinib at $40 \mathrm{mg}$ oral daily. She had a severe rash and diarrhea, as a result dose was reduced to $30 \mathrm{mg}$ daily, which she tolerated well. After 6 months of treatment, she had a good clinical response with improvement in Karnofsky performance status from $50 \%$ to $70 \%$.

Most recent physical examination on afatinib $30 \mathrm{mg}$ daily, was remarkable for obvious $2.0 \times 2.0 \mathrm{~cm}$ moderately firm nodule, on the right side just below her cricothyroid, which was smaller and softer compared to prior examination. There was no significant lymphadenopathy. Sensation was decreased to light touch in bilateral upper extremities, similar compared to prior. Motor strength was noted to be $4 / 5$ bilaterally in the upper extremities, and 5/5 in the bilateral lower extremities. Range of motion of left shoulder was limited in abduction, while that of right shoulder was normal. Cranial nerves and gait were also noted to be normal. Repeat cervical spine MRIs showed no further disease progression. 


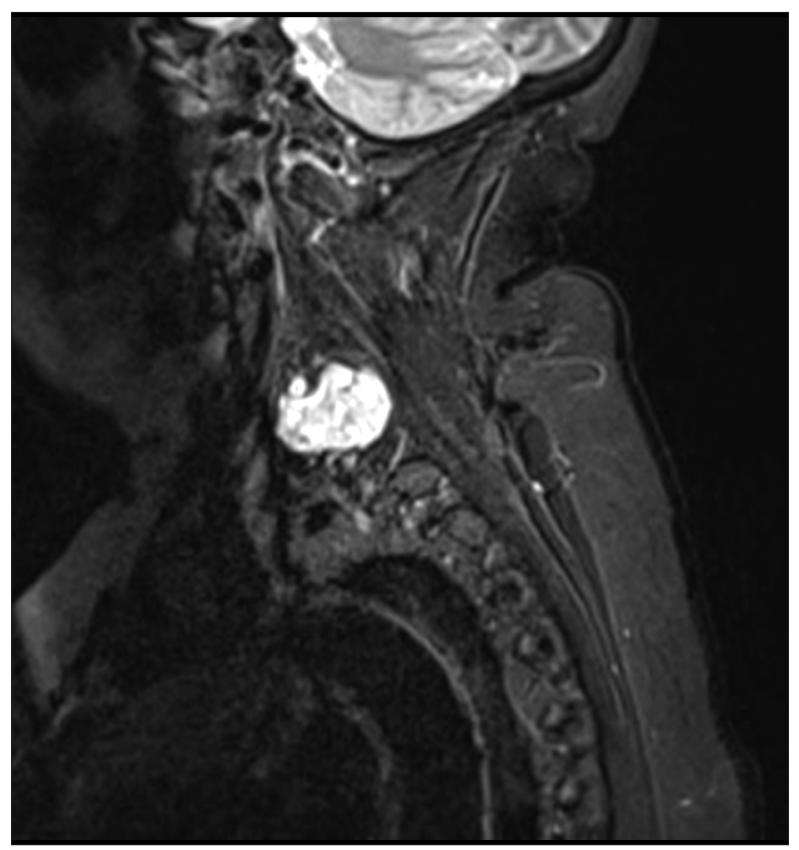

Figure 3. Magnetic resonance imaging cervical spine with and without intravenous contrast. Sagittal STIR image demonstrates a large left paraspinal mass arising from the lateral $\mathrm{C} 5$ body with heterogeneously hyperintense signal.

\section{Discussion}

Chordomas are primary bone tumors, which arise from notochord remnants along the axial skeleton. Brachyury, a transcription factor encoded by $T$ gene, is involved in notochord development and maintenance [3]. Studies have shown that aberrations in T gene play an integral role in initiating tumorigenesis. This tumor occurs largely sporadically, but, $\mathrm{T}$ gene duplications found in family members has provided evidence that there may be a component of genetic predisposition to the development of chordomas [4].

Chordomas account for $1 \%$ to $4 \%$ of all bone tumors and $0.5 \%$ of intracranial neoplasms, with incidence reported at 0.08/100 000; $0.1 / 100000$ in males and 0.06/100 000 in females [1,2]. Usually men in their $50 \mathrm{~s}$ and $60 \mathrm{~s}$ are affected while children and women contribute to a smaller percentage. Chordomas can affect any part of the axial skeleton, with $32 \%$ cases occurring intracranially, $29.2 \%$ in the sacrum, and $32.8 \%$ affecting the rest of the spine [5]. Metastasis affected around $30 \%$ to $40 \%$ of all cases, with lungs, liver, lymph nodes and cerebrospinal (CSF) as the commonly reported sites [6]. Survival is modest ranging from $50 \%$ to $68 \%$ at 5 years and $28 \%$ to $40 \%$ at 10 years [7].

Histologically, chordomas consist of fibrous tissue separating chords of tumor from mucoid matrix. There are 3 histological subtypes: classical, chondroid and de-differentiated, with the latter being the most aggressive and affecting mostly children [6]. Immuno-histological markers are needed to establish diagnosis and to differentiate it from other tumors like chondrosarcoma. Chordoma stains positive for nuclear brachyury along with S-100, epithelial membrane antigen, vimentin, and cytokeratin [8].

Chordoma is a slow growing tumor, which is locally invasive and destructive, eventually leading to compression of surrounding structures including vessels and nerves. Symptoms depend on the site involved and size of the tumor. Intracranial tumors can produce headaches, defects in the visual field, dysphagia, cranial neuropathies and radiculopathies; cranial nerve VI is commonly affected. Sacral location can result in back pain, dysfunction of sphincter, radiculopathies, and motor weakness of lower extremities $[9,10]$.

A diagnosis begins with radiological imaging. X-ray can show bone erosions with irregular calcium foci along with a lytic lesion. Computed tomography scan typically shows a low attenuation, hyperdense soft tissue mass with calcification, with lytic lesions and a locally destructive pattern. MRI is considered superior to other imaging modalities and lesions appear hypodense on T1 flair. Heterogenous enhancement is visualized with gadolinium use. While on T2 images lesion appears hyper dense in comparison [9].

The treatment consists of complete surgical resection with negative margins, however, despite aggressive resection recurrence is common. An incomplete surgical resection, skullbased location, advanced age, and large size and tumor necrosis are associated with poor prognosis [11-13]. A complete resection is often not possible given the proximity to critically important structures, hence, an attempt is made to resect as much tissue as possible. For intra cranial lesions, endoscopic endo-nasal trans-sphenoid approach is the most common approach as it is minimally invasive and equally effective compared to other approaches. Other procedures include transmandibular, subtemporal preauricular, lateral transcondylar, and transcochlear approaches [5,14-16].

Aggressive surgical resection is often combined with radiation therapy with target dose of greater than $60 \mathrm{~Gy}$. This leads to better disease control and reduced local recurrence. Proton beam therapy is preferred over photon therapy, for its straighter trajectory. This results in radiation primarily targeting the lesion, hence minimizing damage to surrounding critical structures $[17,18]$.

There is no established definitive treatment outside of surgery. Chemotherapy has not shown to be effective, hence the focus has shifted towards investigating molecular targeted therapy as a treatment modality $[19,20]$. 
Tyrosine kinase receptor (TKR) expression with aberrations in the downstream signaling molecules is associated with tumorigenesis. Many receptors, genetic alterations, chromosomal abnormalities, and molecular pathways have recently been identified for better an understanding of pathogenesis and management of chordomas. TKRs, including, but not limited to, epidermal growth factor (EGFR), platelet derived growth factor receptor- $\alpha$, platelet derived growth factor receptor $\beta$ amongst many others have been implicated in the development of chordomas either via overexpression or activation [21,22]

Downstream molecular pathway abnormalities associated with this tumor include Akt/phosphoinositide-3-kinase (PI3K)/mammalian target of rapamycin (mTOR), mitogen-activated protein kinase (MAPK)/extracellular signal-regulated kinase (ERK) pathway, along with Janus kinase (JAK)/signal transducer, activation of transcription (STAT) pathway, and abnormalities in retinoblastoma pathway $[22,23]$.

Extensive evidence is available in the current literature in the form of published case reports, retrospective case series, and some phase 2 clinical trials supporting the utility and effectiveness of various receptor inhibitor like imatinib, cetuximab, gefitinib erlotinib, lapatinib, sirolimus, dasatinib, and bevacizumab.

Imatinib (PDGFR, C-KIT, ABL) is one of most widely studied receptor inhibitor in the management of chordomas in a study that involved 50 patients. One patient (2\%) achieved partial response, while 35 patients (70\%) had stable disease [24]. Sunitinib was studied in a phase II trial that included 9 patients with chordoma. In this study, 4 patients had stable disease for at least 16 weeks [25]. The efficacy of imatinib and sirolimus combination was studied in 10 patients with advanced chordoma, 1 patient achieved a partial response, 7 patients had stable disease, while 1 patient had progressive disease [26].

A phase II trial exploring sorafenib in the treatment of locally advanced and metastatic chordoma demonstrated a 9-month progression-free rate of $73 \%$ [27]. In a phase 2 trial, lapatinib was tested in 18 patients with EGFR positive advanced chordoma. The study showed partial responses in 6 patients (33.3\%), stable disease in 7 patients (38.9\%) and a median progression free survival of 6 months as per the Choi criteria. The median progression free survival was 8 months as per the response evaluation criteria in solid tumors (RECIST criteria). Stachiiotti et al. also studied the efficacy of lapatinib in a phase II trial including 18 patients with advanced, progressive EGFR positive chordoma and demonstrated that 33\% patients ( 6 out of 18 patients) had a partial response and $38.9 \%$ patients (7 out of 18 patients) had stable disease [28]. The response to dasatinib was studied on 32 patients with chordoma in another phase II clinical trial and demonstrated that $54 \%$ patients had progression free survival (at 6 months) [29].
Bevacizumab in combination with erlotinib produced stable disease at 2 to 4 and a half year in a case series of 3 patients reported in Sweden [30]. A patient with recurrent lumbar chordoma despite previous gross resection with wide margins and adjuvant radiation treatment developed durable response after being treated with pazopanib for over 15 months [31]. The benefit was also observed in a case series where a patient on sunitinib had a partial response lasting 27 weeks and 4 patients on pazopanib had a median progression free interval of 8.5 months [32].

Clinical trials are being conducted to investigate the role of afatinib, another EGFR inhibitor in chordomas. Published data comparing various EGFR inhibitors has suggested that afatanib is a promising agent with superior results, while lapatinib has been the least effective. Cell lines found to be most sensitive to afatinib activity lacked STK 33 mRNA/protein levels, which could potentially lead to STK 33 being used as biomarker to predict tumor responsiveness to afatinib [33].

Other genetic abnormalities consist of SMARC B1/INII loss, aberrations in micro RNA, in particular miR-1, miR-206, and miR155 amongst several others, and gains or losses related to certain chromosomes [34,35]. In particular, loss of $1 p 36$ has been associated with a poor prognosis. Loss of $9 p$ region leads to CDK4/6 activation via the retinoblastoma pathway and contributes to tumor progression. Palbociclib is being studied as a CKD4/6 inhibitor for chordoma treatment [36].

A number clinical trials are ongoing and studying the effectiveness of afatinib, LBH589, palbociclib, the combination of imatinib with everolimus (Eudra CT 2010-021755-34), the use of oncolytic bacteria, a EZH2 inhibitor, tazemetostat, in adult chordoma patients who have INII negative tumors.

Furthermore, immunotherapy has gained popularity for cancer treatment as it has shown promising results in the management of several malignancies, and now its usefulness is being explored for chordomas. Although originally programmed death ligand 1-2 were not thought to be expressed on chordoma cells, they have now been identified on infiltrating lymphocytes and can be induced by inflammation [37,38]. Ongoing trials are investigating nivolumab and ipilimumab, nivolumab with radiation, and nivolumab with or without stereotactic radiation therapy. Also being explored are irradiated natural killer (NK) cells in combination with cetuximab, as radiation exposure is believed to increase antibody dependent cytotoxicity of cetuximab by increasing the expression of CD16 alleles on NK cells [39].

Chimeric antibody receptor (CAR) therapies have also been developed against chondroitin sulfate proteoglycan-4 (CSPG-4), a cell surface molecule associated with a poor prognosis and 
metastasis. This also paves the way for more upcoming treatment options [40,41].

Vaccination targeting brachyury is also under investigation, as brachyury is known to play a significant role in the pathogenesis of chordomas. In a recent trial investigating the efficacy of a yeast-based vaccine, 11 patients were evaluated and, after 3 months 8 patients had stable disease [42]. Currently trials on $\mathrm{Gl}-6301$ vaccine in combination with radiotherapy are underway, because radiation is thought to increase infiltration by inflammatory cells along with increased expression of $\mathrm{MHC}$ peptides. This increases T cell activity against cancer cells (NCT02383498).

Our case highlights the effectivity of afatinib as a treatment modality for chordoma, that recurs despite multiple surgical resections, radiation, and use of imatinib. Disease control lasted for at least at 6 months. She had a good clinical response with improvement in Karnofsky performance status from $50 \%$ to $70 \%$ and repeat cervical spine MRIs showed no further disease progression.

\section{References:}

1. Healey JH, Lane JM: Chordoma: A critical review of diagnosis and treatment Orthop Clin North Am, 1989; 20(3): 417-26

2. Committee of Brain Tumor Registry of Japan: Report of brain tumour registry of Japan (1984-2000). Neurol Med, 2009; 49 Suppl.: PS1-96

3. Vujovic S, Henderson S, Presneau $\mathrm{N}$ et al: Brachyury, a crucial regulator of notochordal development, is a novel biomarker for chordomas. J Pathol, 2006; 209(2): 157-65

4. Yang XR, Ng D, Alcorta DA et al: T (brachyury) gene duplication confers major susceptibility to familial chordoma. Nat Genet, 2009; 41(11): 1176-78

5. McMaster ML, Goldstein AM, Bromley CM et al: Chordoma: Incidence and survival patterns in the United States, 1973-1995. Cancer Causes Control, 2001; 12(1): 1-11

6. Fletcher C, Bridge J, Hogendoorn P, Martens F: World Health Organization (WHO) classification of tumours of soft tissue and bone. Pathology and Genetics. 2013

7. Sciubba DM, Chi JH, Rhines LD, Gokaslan ZL: Chordoma of the spinal column. Neurosurg Clin N Am, 2008; 19(1): 5-15

8. Crapanzano JP, Ali SZ, Ginsberg MS, Zakowski MF: Chordoma: A cytologic study with histologic and radiologic correlation. Cancer, 2001 ;93(1): 40-51

9. Khawaja AM, Venkatraman A, Mirza M: Clival chordoma: Case report and review of recent developments in surgical and adjuvant treatments. Pol J Radiol, 2017; 82: 670-75

10. Walcott BP, Nahed BV, Mohyeldin A et al: Chordoma: Current concepts, management, and future directions. Lancet Oncol, 2012; 13(2): e69-76

11. Gay E, Sekhar LN, Rubinstein E et al: Chordomas and chondrosarcomas of the cranial base: Results and follow-up of 60 patients. Neurosurgery, 1995; 36(5): 887-97

12. Bohman L, Koch M, Bailey RL et al: Skull base chordoma and chondrosarcoma: Influence of clinical and demographic factors on prognosis: A SEER analysis. World Neurosurg, 2014; 82(5): 806-14

13. Di Maio S, Temkin N, Ramanathan D, Sekhar LN: Current comprehensive management of cranial base chordomas: 10-year meta-analysis of observational studies. J Neurosurg, 2011; 115(6): 1094-105

14. Fraser JF, Nyquist GG, Moore $\mathrm{N}$ et al: Endoscopic endonasal transclival resection of chordomas: Operative technique, clinical outcome, and review of the literature. J Neurosurg, 2010; 112(5): 1061-69

15. Llorente JL, Obeso S, Rial JC et al: Surgical treatment of clival chordomas. Acta Otorrinolaringologica (English Edition), $2010 ; 61(2)$ : 135-44

\section{Conclusions}

Due to the rarity of chordomas, limited trials have been carried out. As a result, there is lack of standardized systemic and effective therapy. At present, enbloc resection with possible radiation therapy remains the only effective treatment option, and there is significant morbidity associated with the tumor. There is optimism regarding development of standardized therapies, however, that will require more funding and internal collaborations that are carried out on a larger scale. Our patient demonstrated that afatinib should be studied for progressive chordoma, given positive response observed in our patient.

\section{Conflict of interest}

None.

16. Jahangiri A, Jian B, Miller L et al: Skull base chordomas: Clinical features, prognostic factors, and therapeutics. Neurosurg Clin N Am, 2013; 24(1): 79-88

17. Jawad MU, Scully SP: Surgery significantly improves survival in patients with chordoma. Spine, 2010; 35(1): 117-23

18. Frank SJ, Selek U: Proton beam radiation therapy for head and neck malignancies. Curr Oncol Rep, 2010; 12(3): 202-7

19. Colia V, Stacchiotti S: Medical treatment of advanced chordomas. Eur Cancer, 2017; 83: 220-28

20. Fleming GF, Heimann PS, Stephens JK et al: Dedifferentiated chordoma Response to aggressive chemotherapy in two cases. Cancer, 1993; 72(3): 714-18

21. Akhavan-Sigari R, Abili M, Gaab M et al: Immunohistochemical expression of receptor tyrosine kinase PDGFR- $\alpha$, c-Met, and EGFR in skull base chordoma. Neurosurg Rev, 2015; 38(1): 89-99

22. Fasig J, Dupont W, LaFleur B et al: Immunohistochemical analysis of receptor tyrosine kinase signal transduction activity in chordoma. Neuropathol Appl Neurobiol, 2008; 34(1): 95-104

23. Wang L, Zehir A, Nafa $K$ et al: Genomic aberrations frequently alter chromatin regulatory genes in chordoma. Genes Chromosomes Cancer, 2016; 55(7): 591-600

24. Stacchiotti $S$, Longhi A, Ferraresi V et al: Phase II study of imatinib in advanced chordoma. J Clin Oncol, 2012; 30(9): 914-20

25. George S, Merriam P, Maki RG et al: Multicenter phase II trial of sunitinib in the treatment of nongastrointestinal stromal tumor sarcomas. J Clin Oncol, 2009; 27(19): 3154-60

26. Stacchiotti S, Marrari A, Tamborini E et al: Response to imatinib plus sirolimus in advanced chordoma. Ann Oncol, 2009; 20(11): 1886-94

27. Bompas E, Le Cesne A, Tresch-Bruneel E et al: Sorafenib in patients with locally advanced and metastatic chordomas: A phase II trial of the French Sarcoma Group (GSF/GETO). Ann Oncol, 2015; 26(10): 2168-73

28. Stacchiotti S, Tamborini E, Lo Vullo $S$ et al: Phase II study on lapatinib in advanced EGFR-positive chordoma. Ann Oncol, 2013; 24(7): 1931-36

29. Schuetze SM, Bolejack V, Choy E et al: Phase 2 study of dasatinib in patients with alveolar soft part sarcoma, chondrosarcoma, chordoma, epithelioid sarcoma, or solitary fibrous tumor. Cancer, 2017; 123(1): 90-97

30. Asklund T, Sandström M, Shahidi S et al: Durable stabilization of three chordoma cases by bevacizumab and erlotinib. Acta Oncol, 2014; 53(7): 980-84 
31. Ribeiro MFSA, de Sousa MC, Hanna SA et al: Tumor reduction with pazopanib in a patient with recurrent lumbar chordoma. Case Rep Oncol Med, 2018; 2018: 4290131

32. Lipplaa A, Dijkstra S, Gelderblom H: Efficacy of pazopanib and sunitinib in advanced axial chordoma: a single reference centre case series. Clin Sarcoma Res, 2016; 6(1): 19

33. Magnaghi $P$, Salom B, Cozzi L et al: Afatinib is a new therapeutic approach in chordoma with a unique ability to target EGFR and brachyury. Mol Cancer Ther, 2018; 17(3): 603-13

34. Antonelli M, Raso A, Mascelli S et al: SMARCB1/INI1 involvement in pediatric chordoma. Am J Surg Pathol, 2017; 41(1): 56-61

35. Longoni $M$, Orzan $F$, Stroppi $M$ et al: Evaluation of 1p36 markers and clinical outcome in a skull base chordoma study. Neurooncology, 2008; 10(1): 52-60

36. von Witzleben A, Goerttler LT, Marienfeld R et al: Preclinical characterization of novel chordoma cell systems and their targeting by pharmocological inhibitors of the CDK4/6 cell-cycle pathway. Cancer Res, 2015; 75(18): 3823-31
37. Mathios D, Ruzevick J, Jackson CM et al: PD-1, PD-L1, PD-L2 expression in the chordoma microenvironment. J Neurooncol, 2015; 121(2): 251-59

38. Feng Y, Shen J, Gao Y et al: Expression of programmed cell death ligand 1 (PD-L1) and prevalence of tumor-infiltrating lymphocytes (TILS) in chordoma. Oncotarget, 2015; 6(13): 11139-49

39. Fujii R, Schlom J, Hodge JW: A potential therapy for chordoma via antibodydependent cell-mediated cytotoxicity employing NK or high-affinity NK cells in combination with cetuximab. J Neurosurg, 2018; 128(5): 1419-27

40. Schoenfeld AJ, Wang X, Wang Y et al: CSPG4 as a prognostic biomarker in chordoma. Spine J, 2016; 16(6): 722-27

41. Beard RE, Zheng Z, Lagisetty $\mathrm{KH}$ et al: Multiple chimeric antigen receptors successfully target chondroitin sulfate proteoglycan 4 in several different cancer histologies and cancer stem cells. J Immunother Cancer, 2014; 2(1): 25

42. Heery $C R$, Singh $B H$, Rauckhorst $M$ et al: Phase I trial of a yeast-based therapeutic cancer vaccine (GI-6301) targeting the transcription factor brachyury. Cancer Immunol Res, 2015; 3(11): 1248-56 